\title{
The Beam Current Considerations in SEM Accordance to Mirror Effect Phenomenon
}

\author{
Hassan N. Al-Obaidi, Imad H. Khaleel \\ Physics Department, College of Education, Al-Mustansiriah University, Baghdad, Iraq \\ E-mail address: hassanmail2006@yahoo.com
}

\begin{abstract}
A theoretical investigation have been presented to exploring the influence of electrons beam current on the electron mirror image deduced inside the scanning electron microscope (SEM). A rough mathematical expression for the electric potential that associated with electron beam is derived. The results clearly shows that the beam current could be used to enhance or conversely deteriorate the phenomena of mirror effect. So this work procedure may consider to be tool controllable of this phenomena for investigation purposes.
\end{abstract}

Keywords: Scanning Electron Microscope; Electron Beam Current; Electron Mirror Images

\section{INTRODUCTION}

Today, the charged particle devices ( especially the scanning electron microscope and focused ion beam (FIB)) are considered to be an important tools for collecting data details for materials in the range of nano-scale. Actually, the technical characteristics of an SEM are determined by the parameters of its electron beam. Anyway, the most important parameters of the electron beam are the size (diameter) of the focused electron beam, angles of its convergence and divergence, and the depth of its focusing [1]. This information is important both for the development and upgrading of the construction of an SEM, and for the use of the microscope in scientific research and industries. Information regarding the geometrical characteristics of the electron beam is of particular importance in connection with the development of new methods for measurements of linear dimensions of the relief elements in the micro- or nanometer range, being on a surface of a solid [2].

In fact, beam current is one of the most important parameters that influence the electron beam diameter [3], and so it is a very important instrumental parameter. Strictly speaking low beam current and small spot size is usually required for high resolution, while high beam current and large spot diameters is ultimately useful for good contrast [4]. So, condenser lens in SEM should be operate in compensate way such that it balance these two parameters according to specific application.

What have mentioned in the last paragraph is valid well for the usual use of SEM to imaging conductive samples or either metal-coated insulator samples. However, when a sample for a dielectric material (or either not grounded conducting sample) is adopted for 
inspection by means of SEM or FIB, the mirror effect come to be occurs inside the SEM chamber, see for example the references [5] and [6].

Substantially, this phenomenon arises due to the accumulation of charges, electrons in SEM [7] and ions in FIB [8], at the sample surface during the irradiation process. Consequently, an electric potential begin to growth over the irradiated region up to the state of saturation [9]. This potential in turn made the probing electrons (electron of lower potential) to reflecting-back towards the upper chamber space. Therefore, the screen of SEM apparatus will reveals an image that refers to the ceiling of SEM chamber rather than the sample. Electron mirror images have been gained more advertency since its invention in the seventeenths of last century. Iit is now attracting a great deal of attention, especially when some authors argued that it can used as a tool to determine some of the characteristics of insulator materials [9 and 10].

Indeed many operation parameters controls the image which deduced by means of mirror effects. Among most of them are the irradiation (potential, current and time) [11,12], scanning (potential [9] and current), working distance [13] and the dielectric constants [10] of the insulator material under consideration. Present work, is mainly concerned with influence of the electron beam current. However, when mirror effect come to be occurs inside the SEM chamber, and the aim is to avoid that, it doesn't important to use low beam current with small spot diameter or vice versa. Thus, in sense of such a framework, present work try to prove that it is possible to eliminate the mirror effect phenomena by increasing the beam current.

\section{THEORETICAL CONCEPTS}

Initially let assume the electrons are distributed uniformly within the beam and so the face of this beam being a disk of radius $R$ that carry charges of amount $Q$, see figure.1. The aim, however, is to find the electric potential $U_{b}(\vec{r})$ at a point $\mathrm{P}$ located at a distance $\vec{r}$ from the beam's disk. According to the basics ideas of electromagnetic, the potential $U_{b}(\vec{r})$ can be represented by the following expression [14];

$$
U_{b}(\vec{r})=\frac{1}{4 \pi \varepsilon_{0}} \int_{S} \frac{\sigma^{\prime}\left(\vec{r}^{\prime}\right) d a^{\prime}}{\left|\vec{r}-\vec{r}^{\prime}\right|}
$$

Where $\sigma^{\prime}\left(\vec{r}^{\prime}\right)$ is the surface charge density embedded in the disk, $d a^{\prime}$ is an infinitesimal area from the disk which bounded by the surface S. Using the cylindrical coordinates and keeping in mind that charges is uniformly distributed across the surface disk, equation (1) can be written as;

$$
U_{b}(\vec{r})=\frac{\sigma^{\prime}}{4 \pi \varepsilon_{0}} \int_{0}^{R} \frac{r^{\prime} d r^{\prime}}{\left|\vec{r}-\vec{r}^{\prime}\right|} \int_{0}^{2 \pi} d \varphi
$$

When the disk being at a right angle with beam axis the last equation becomes; 


$$
U_{b}(\vec{r})=\frac{\sigma^{\prime}}{2 \varepsilon_{0}} \int_{0}^{R}\left(\vec{r}^{2}+r^{\prime 2}\right)^{-1 / 2} r^{\prime} d r^{\prime}
$$

Integration of the last equation is simple, however, the result is;

$$
U_{b}(\vec{r})=\frac{\sigma^{\prime}}{2 \varepsilon_{0}}\left\{\left(\vec{r}^{2}+R^{2}\right)^{1 / 2}-\vec{r}\right\}
$$

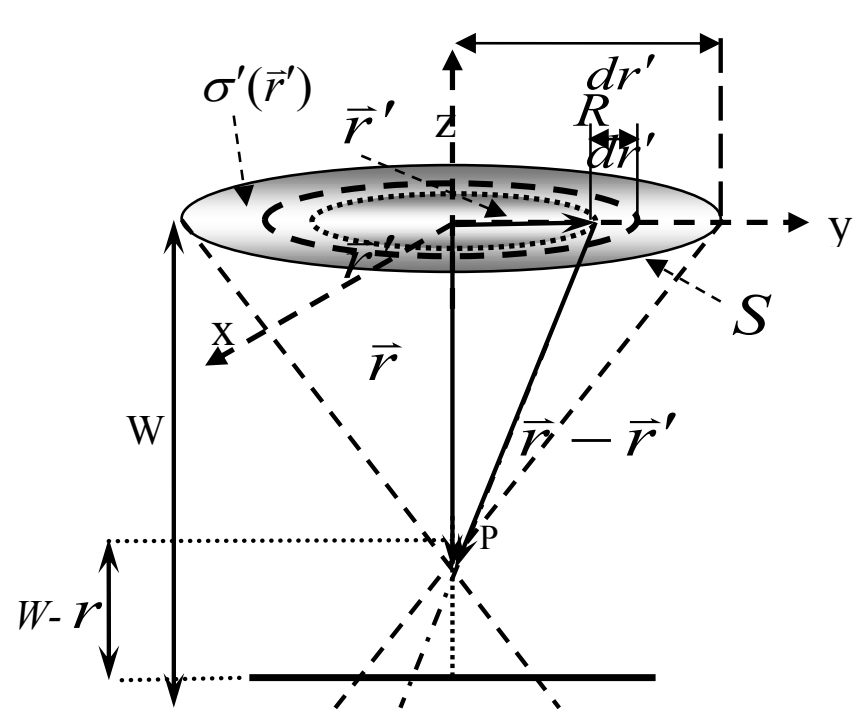

Figure 1. A schematic representation for beam of electrons landing on a sample that is previously irradiated.

Since the surface charge density is assumed to be uniform one may say without caution, that; $\sigma^{\prime}\left(\vec{r}^{\prime}\right)=Q / S$, where $S=\pi R^{2}$. According to the current definition the total electrons $(Q)$, that embedded in the surface $S$, can be expressed in terms of the beam current $(I)$ as follows; $Q=I t$. Where $\mathrm{t}$ is the unit of time per which the electrons $(Q)$ will pass through any point $(x, y, z)$ within the beam space. Thus, equation (4) becomes;

$$
U_{b}(\vec{r})=\frac{I t}{2 \pi \varepsilon_{0} R^{2}}\left\{\left(\vec{r}^{2}+R^{2}\right)^{1 / 2}-\vec{r}\right\}
$$

Since the above manipulation is mainly concerned with finding beam potential along with the beam axis, see Figure.1, the last equation can be written as;

$$
U_{b}(z)=\frac{I t}{2 \pi \varepsilon_{0} R^{2}}\left\{\left(z^{2}+R^{2}\right)^{1 / 2}-z\right\}
$$


Equation (6) reveals that the beam current and beam diameter are the only parameters that influence the potential, that deduced from electrons forms the beam surface, along the distance from the beam surface till the sample.

\section{RESULTS AND DISCUSSIONS}

Figure 2 shows the variation of beam surface potential along with the vertical distance $(W-z)$ for two arbitrary values of the beam current. Where $W$ is the working distance, which taken to be $15 \mathrm{~mm}$, and $z$ refers to the absolute value of the vector $\vec{r}$, see Figure 1 . It is seen that the two potential distributions have a maximum value at the beam surface. However the value of $U_{b}(W-z)$ being decreases as one moves away from the beam surface toward the sample.

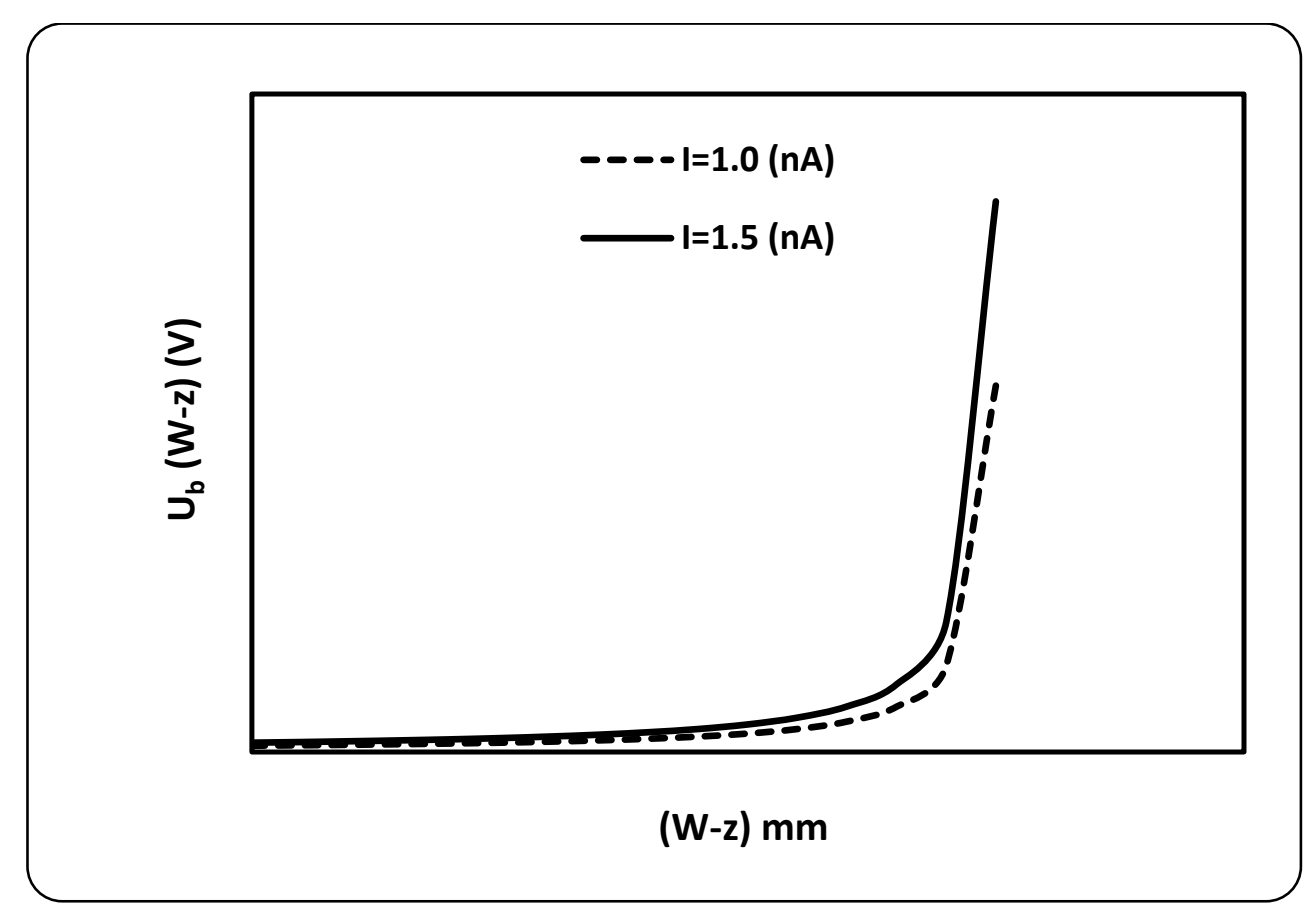

Figure 2. The variation of beam surface potential along the vertical axis $(W-z)$.

Actually the decreases behavior being sharp near the beam surface and gradually decreases when $z$ increases. The reason behind is the inverse proportional of $U_{b}(\vec{r})$ with $\vec{r}$ as one could realized by consulting equation (1). Equivalently it is a direct consequence for the inverse square proportionality associated with Coulomb's law. Strictly speaking, when an electron try to approach the beam surface it will suffer a repulsive force which increases rapidly when electron touch the surface.

Another remarkable result can be record from Figure 2, that when the beam current increased the beam potential increased too along the distance $W-z$. For more clearness the values of beam potential at the terminal points of the vertical distance $(W-z)$ are plotted as a function of beam current in Figure 2. It seen that the potential increases more rapid near the beam surface in comparison with remaining end. 


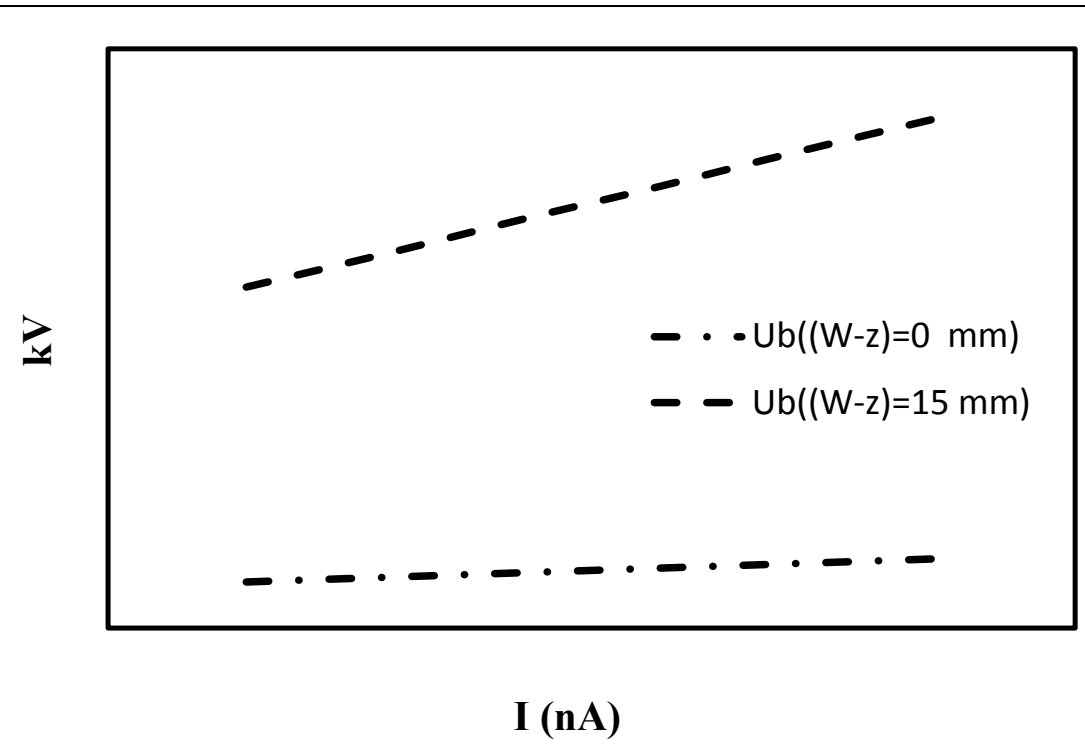

Figure 3. The variation of beam surface potential versus the beam current at the terminal points of vertical axis $(W-z)$.

The increases in beam potential may thought to be a consequence for increasing the number of electrons embedded at beam surface. In fact, such a result is proved in figure.4. Where it is clear that the relation between beam current and the total amount of electrons at beam surface is a linear relationship. So, one can say that the probability for beam of electrons to attack the sample surface increases as the beam current increases too. In other word, ability of beam of electrons to reaching sample surface become height as the condenser lens diaphragm being wider. Therefore, mirror effect can be avoid somehow by increasing beam current.

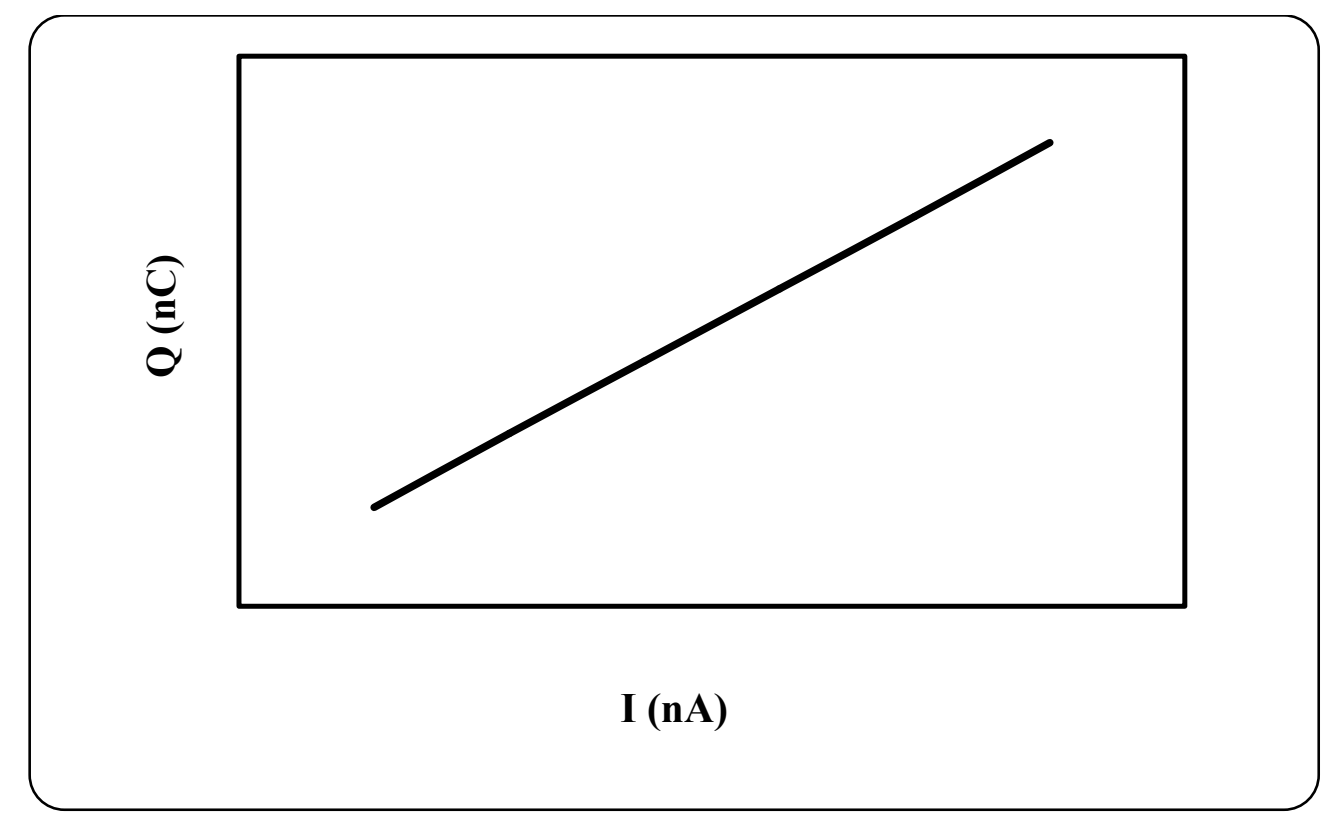

Figure 4. The amount of electrons embedded at beam surface versus the beam current. 


\section{CONCLUSIONS}

One of the most important conclusion remarks of this work is that the beam current could be add to the tools or approaches by means mirror effects could be prevents to be occurs inside the SEM chamber. Conversely, it may adopted to reinforce the phenomena of mirror effects for specific uses of SEM like determination of dielectric constant of insulator materials.

\section{References}

[1] Robert L. E., Scanning electron microscopy and X-ray microanalysis, Prentice-Hall, 1993.

[2] Novikov Y. A., Rakov A. V., Russia, Microelectronics 25(6) (1996) 375-383.

[3] Egerton R.F., Malac P. M., Micron 35 (2004) 399-409.

[4] Chad P., Dale B., Curt P., Phillip R., Microscopy and Analysis 21(5) (2007) 11-13.

[5] Okai N. and Sohda Y., Jap. J. Appl. Phys. 52 (2012) 06FB11.

[6] Ghorbel N., Kallel A., Dammame G., Renoud R., Fakhakh Z., Electrets, ISE-12 $12^{\text {th }}$ International Symposium, (2005) 235-238.

[7] Al-Obaidi H. N., Al-Saymary F. A., Ali A. A., Proc. Summ. Scho., Sep. $8^{\text {th }}$ Oct. $8^{\text {th }}$, Milano, Italy, 2008.

[8] Croccolo F., Riccardi C., J. Microscopy 229(1) (2008) 39-43.

[9] Abbood T. H., "Formal Investigation of the Mirror Effect in SEM." Ph.D. thesis, University of Al-Mustansiriyah, College of Education, Baghdad, Iraq, 2011.

[10] Milani M., Abdul-Wahab H. N., Abbood T. H., Savoia C., Tatti F., Sci., Tech. and Edu. (2010) 1741-1754.

[11] Belhaj M., Jbara O., Odof S., Msellak K., Rau E., Andrianov M., Scanning 88 (2008) 352-374.

[12] Belhaj M., Odof S., Msellak K., Jbara O., J. Appl. Phys. 88(5) (2000b).

[13] Milani M., Savoia C., Bigoni D., Proceedings of ITP2009, Interdisciplinary Transport Phenomena VI: Fluid, Thermal, Biological, Materials and Space Sciences, Volterra, Italy 2009.

[14] Hassan N., Imad H. Khalele, Huda K. Husien, Influnce of Electron Beam Charactaristics on the Electron Mirror Images of SEM, under publication, 2013. 